
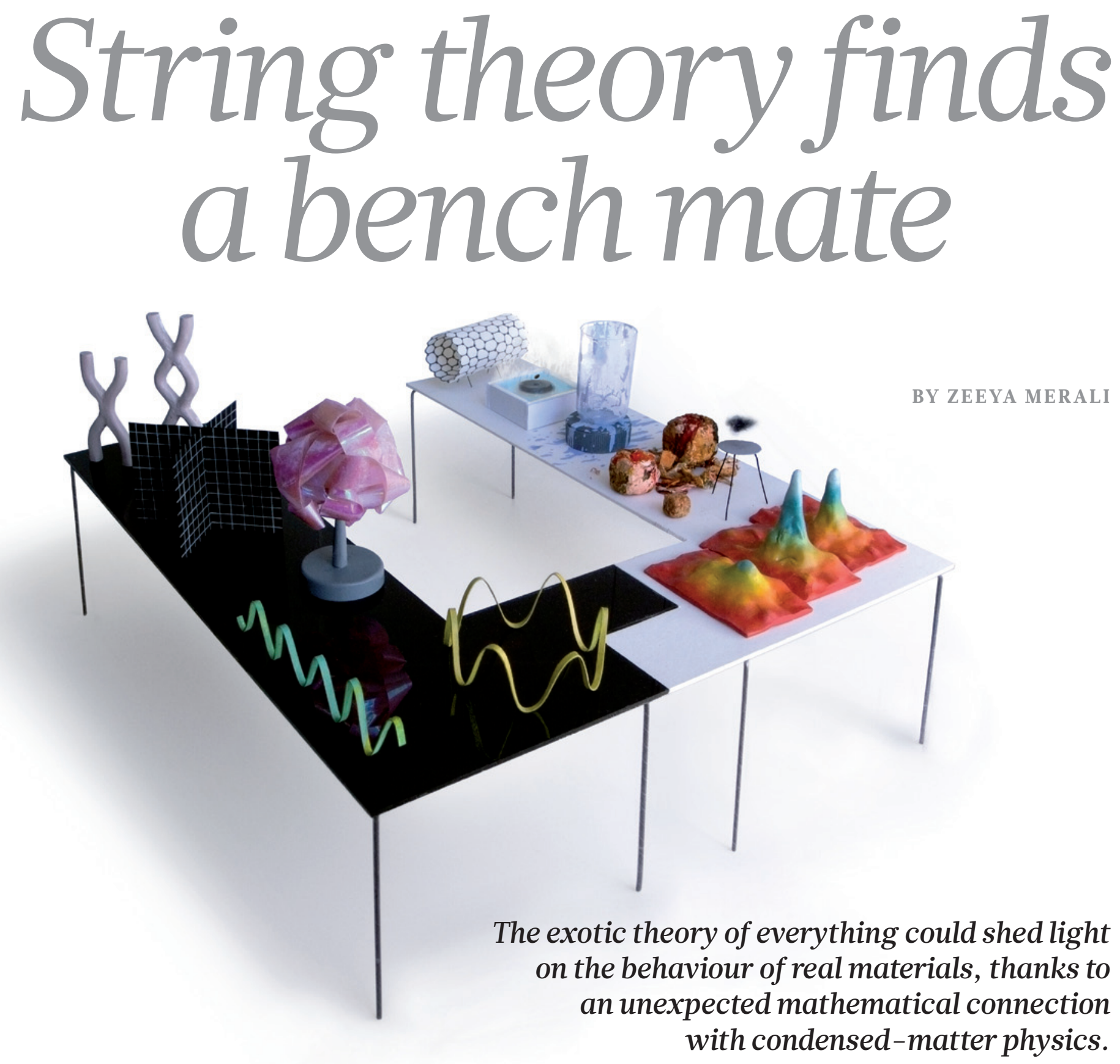

"O n one side," says Jan Zaanen, "you have this refined, almost other-worldly intellectual - the perfectionist obsessed with detail, barely interested in earthly pleasures. On the other, you have the loud, boisterous, sometimes aggressive, business-savvy character who knows how to get his hands dirty."

It might almost be a description of the misfit roommates known on stage, screen and television as The Odd Couple. But Zaanen, a condensedmatter physicist at the University of Leiden in the Netherlands, is actually describing the pairing of two groups of scientists: string theorists, who spend their days pursuing a rarefied, highly mathematical 'theory of everything, and his own colleagues - a considerably more grounded bunch who prefer to focus on how real-world materials behave in the laboratory.

The scientists trying to bridge these disciplines are motivated by the discovery of a startling coincidence: suitably interpreted, the equations of string theory can be a powerful tool for analysing some exotic states of matter, ranging from super-hot balls of quarks and gluons to ultracold atoms. The past year alone has seen at least four international workshops designed to stimulate collaborations across the disciplinary divide, including one hosted by Zaanen in Leiden.

Sceptics still question whether this strange alliance will actually lead to new insights, or whether it is just a marriage of convenience. String theory does hint at the existence of many new states of matter, for example. But those predictions will be difficult to verify, and decisive experimental tests are only now in the planning stages.

For the time being, the advantage to both partners is clear. String theory, long criticized for having lost touch with reality, gets experimental credibility. And condensed-matter physics, never the media darling that string theory has been, gets a new mathematical tool - and a chance to bask in new-found glamour.

The match-making began a dozen years ago with the reunion of Dam Thanh Son and Andrei Starinets, who had been undergraduates and dorm-mates at Moscow State University in the 1980s. The friends had lost touch with each other when they left Russia after the fall of communism in 1991. But in 1999, Son got a job at Columbia University 
in New York City, and heard that Starinets was doing a PhD in string theory just a few kilometres away at New York University. So Son went to pay Starinets a visit.

Collaboration was the farthest thing from his mind. String theory is mathematically rich and has an undeniable aesthetic appeal. But it is all about what physics might be like at scales of $10^{-35}$ metres - the idea being that seemingly point-like elementary particles such as quarks and electrons will actually turn out to be tiny, vibrating threads of energy when viewed at such scales. But these strings would be about 20 orders of magnitude smaller than a proton, putting the theory hopelessly beyond the reach of any direct experimental test. Son's speciality, by contrast, was firmly rooted in experiment: he was trying to understand the properties of quark-gluon plasmas, the short-lived, super-hot fireballs that form when heavy nuclei such as gold are smashed together in accelerators. All this stringy stuff seemed utterly alien.

Except that, when Son saw the string-theory calculations that Starinets had been working on with fellow PhD student Giuseppe Policastro, he recognized the equations as the same ones he had been using to analyse the plasma.

Son immediately had to know what was going on, and Starinets began to explain. Starinets and Policastro had been working on an idea proposed in 1997 by Juan Maldacena, a physicist at Harvard University in Cambridge, Massachusetts. Maldacena, now at the Institute for Advanced Study in Princeton, New Jersey, had realized that string theory predicts a mathematical equivalence between two hypothetical universes, one of which would be similar to our own. It would have the same three dimensions of space and one dimension of time, for example, and be filled with much the same types of elementary particle, which would, in turn, obey familiar-looking (to physicists) quantum-field equations. But it would not contain strings - or gravity.

The other universe would be the opposite: it would contain both strings and gravity - indeed, the gravity could get strong enough to form black holes - but no elementary particles. It would also have an additional dimension of space.

Maldacena's insight was simple, if audacious: take any process involving particles and fields in the first universe, he said, and it could equally well be described as a process involving gravity, black holes and strings in the second universe - and vice versa ${ }^{1}$. The equations might look very different. But the fundamental physics would be exactly the same.

That was why Son was seeing quark-gluon equations in a stringtheory calculation, Starinets explained: they were the three-dimensional equivalent of the gravitational fields that he and Policastro had been studying in the four-dimensional universe.

\section{Marriage of convenience}

All this jumping back and forth between universes was weird even by string-theory standards (and even weirder for non-string theorists, as Maldacena had showed that the mapping worked not just between three and four dimensions of space, but also between four and five, five and six and so on). But as Son and Starinets talked, they began to see that Maldacena's mapping might be a powerful problem-solving strategy. They could start with a messy set of quantum-field calculations in our real, three-dimensional world - the quark-gluon plasma equations, say - then map those into the four-dimensional world, in which the equations tend to be much easier to solve. Then they could map the results back to the three-dimensional world and read off the answer.

It worked. "We turned the calculation on its head to give us a prediction for the value of the shear viscosity of a plasma," says Son, referring to a key parameter of the quark-gluon fireball2. "A friend of mine in nuclear physics joked that ours was the first useful paper to come out of string theory," he says.

In 2008, the team's predictions were confirmed ${ }^{3}$ at the Relativistic Heavy Ion Collider at Brookhaven National Laboratory in Upton, New York. "These were strong quantitative results, and they still stand today as the best results achieved by the programme to relate string theory to experiment,"

says Steve Gubser, a string theorist at Princeton University, and one of the early champions of applying the principle to real-world problems.

The team's success also caught the attention of Subir Sachdev, a condensed-matter theorist at Harvard. Just as Son had seen a plasma reflected back at him in Starinets' equations, Sachdev saw quantum critical-phase transitions - the changes of state that occur in materials when they near absolute zero, when quantum-mechanical effects begin to dominate. "They were using different words," he says, "but it was the same physics."

Sachdev hoped that Maldacena's idea could provide him and his fellow theorists with some much-needed help in exploring this chilly realm. Over the decades, experimentalists had discovered a long list of exotic, quantum-dominated states - including superconductors that allow current to flow without resistance; superfluids that have no viscosity and can creep up the walls of beakers; Bose-Einstein condensates made up of atoms moving in step like a single 'super atom'; and 'strange' metals that behave in ways subtly different from ordinary metals. But physicists still have no way to predict what will turn up in the lab next. "We can't even answer the fundamental question of how many phases of matter exist," says Sean Hartnoll, a string theorist at Stanford University in California.

Sachdev's first efforts to apply Maldacena's idea to laboratory materials had resulted in two papers he co-authored in 2007, one with Son and his colleagues ${ }^{4}$, and another with a team that included Hartnoll ${ }^{5}$. Since then, Sachdev and his collaborators have built up a recipe for mapping the conductivity of strange metals into the properties of black holes in the string theorists' four-dimensional universe - a strategy that string theorist John McGreevy at the Massachusetts Institute of Technology in Cambridge ${ }^{6}$ and others are also pursuing. These groups get answers that broadly reproduce the peculiar low-temperature behaviour of the metals. They have also mapped the behaviour of four-dimensional black holes in string theory to the conditions at which many materials will change phase into states other than the familiar solid, liquid and gas ${ }^{7}$. "We now have a whole new hammer for attacking the problems I have been working on for 20 years," says Sachdev (see 'An unexpected link').

Sachdev's involvement, in turn, has helped to ignite the interest of other condensed-matter physicists. "A lot of us got into this field because of the force of Subir's personality and his reputation - we realized that if he was taking this seriously, maybe we should too," says Andrew Green, a condensed-matter physicist at the University of St Andrews, UK, who co-organized a workshop on the correspondence at Imperial College London in January.

The condensed-matter results also got the string theorists excited eventually. The field had been generally unenthusiastic about following up on the quark-gluon plasma calculations, says Clifford Johnson, a string theorist at the University of Southern California in Los Angeles. And at least part of the reason, he suspects, was a bias against sullying string theory's purity. "There was a snobbery among some towards what was termed 'mere applications'," he says.

But in 2006, string theory took a public battering in two popular books: Not Even Wrong by Peter Woit, a mathematician at Columbia, and The Trouble With Physics by Lee Smolin, a physicist at the Perimeter Institute for Theoretical Physics in Waterloo, Canada. Both books excoriated the theory's isolation from experiment.

"It's hard to say whether the interest in condensed-matter applications is a direct response to those books because that's really a psychological question," says Joseph Polchinski, a string theorist at the Kavli Institute for Theoretical Physics in Santa Barbara. "But certainly string theorists started to long for some connection to reality."

The condensed-matter partnership seemed perfect for that. If nothing else, it promised to make a virtue out of string theory's embarrassment of riches - the roughly $10^{500}$ solutions to its basic equations, each of which describes a possible universe with its own size, shape, dimensionality and physical laws. Through Maldacena’s idea, says

$\rightarrow$ NATURE.COM Hear more about this story in our podcast: go.nature.com/jnpege string theorist Jerome Gauntlett at Imperial College London, "each solution can be expressed in the countless materials yet to be discovered". 


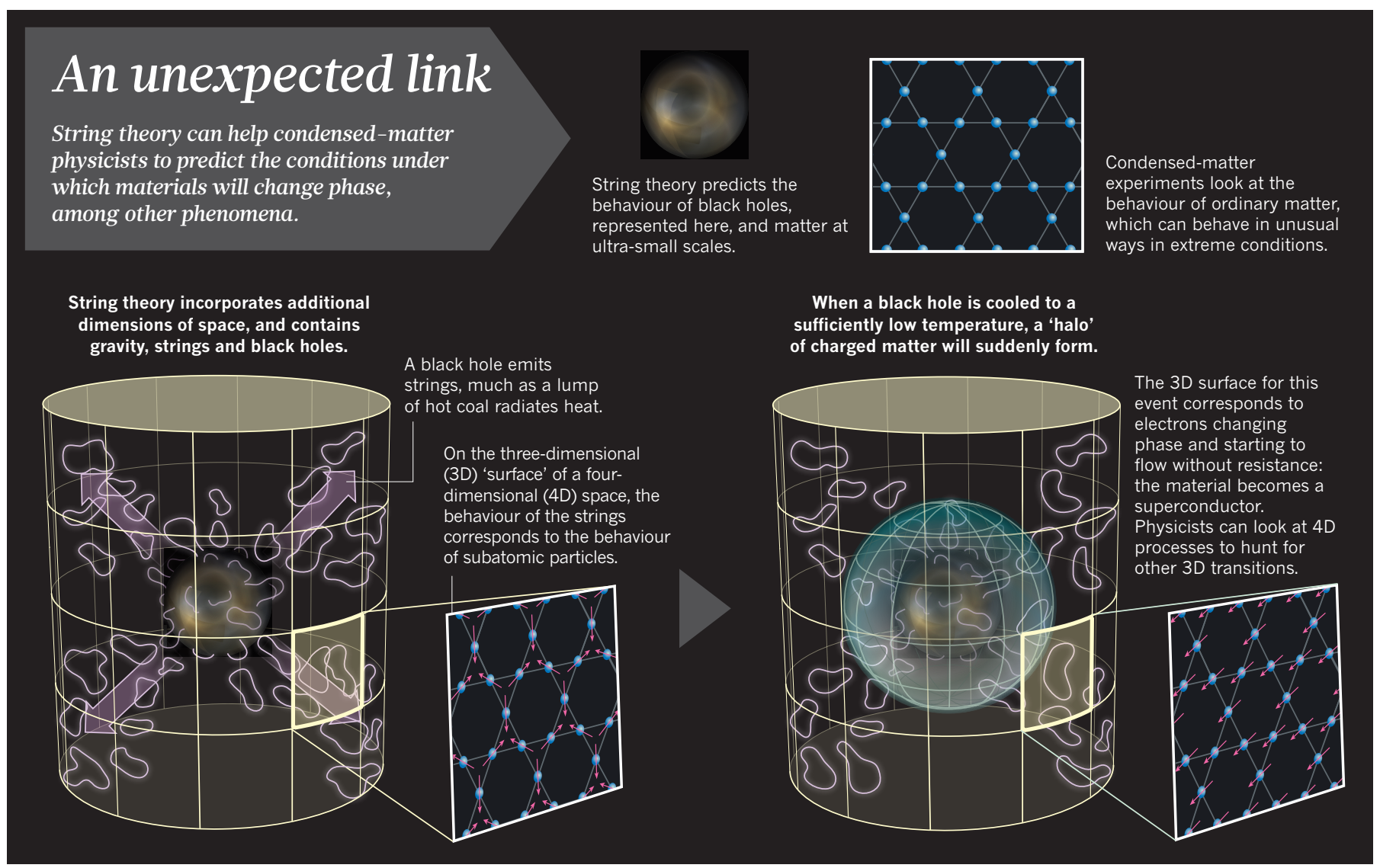

The rewards are mutual, says Zaanen. "If I talk about superconductors and black holes in a colloquium, folk are attracted to it like bees to honey," he says. "It's now bringing young blood to condensed-matter physics, as their first choice."

The flurry of workshops promoting the partnership have been very productive, agrees Polchinski. He co-organized a meeting at the Kavli Institute last year that sparked seven new collaborations, and he is running another from August through November of this year. "It is unique to try to actively make two groups of physicists collaborate so quickly - I haven't personally seen any other similar drive over the course of my career," says Gauntlett, who co-organized the London workshop.

Just as with the fictional odd couple, however, this partnership still has plenty of friction. Everyone agrees, for example, that condensedmatter physicists are much more hesitant about pairing up than their string-theory counterparts. "I have been remarkably unsuccessful at getting condensed-matter physicists to let string theorists speak at their big meetings," says Zaanen. "They fear that they will need to learn string theory to talk to them. It's as though I am asking them to have coffee with aliens."

Polchinski admits that the condensed-matter sceptics have a point. "I don't think that string theorists have yet come up with anything that condensed-matter theorists don't already know," he says. The quantitative results tend to be re-derivations of answers that condensed-matter theorists had already calculated using more mundane methods.

To make matters worse, some of the testable predictions from string theory look a tad bizarre from the condensed-matter viewpoint. For example, the calculations suggest that when some crystalline materials are cooled towards absolute zero, they will end up in one of many lowestenergy ground states. But that violates the third law of thermodynamics, which insists that these materials should have just one ground state. "That's the gorilla in the room that should be keeping people awake at night," says Gubser.

To win over sceptics, theorists are busily searching for testable predictions that will lead to killer evidence that the collaborations are worthwhile. Gauntlett's group, and others, are hunting for black-hole configurations in the string-theory universe that map to undiscovered phase transitions ${ }^{8}$. The trick is to figure out which materials might exhibit those transitions. "Right now that involves going round asking, 'have you seen something like this?', says Gauntlett. "But the hope is that as techniques advance, experimenters will be able to engineer materials with the properties we predict."

Sachdev is applying string theory to an existing challenge: calculating how conductance should change with temperature as ultracold atoms transition from a superfluid state to an insulating one $e^{7}$. He thinks that it should be possible to test his predictions in the next couple of years.

Even if the programme is successful, there are limits to how much the relationship can benefit either partner. String theory can offer a handbook of properties to look for, and predictions for how they should change in experiments, says Green. But it will never be able to provide a theory of how these properties emerge from the behaviour of electrons. Similarly, experimental verification of string theory's predictions about condensed matter will not prove that strings themselves are an accurate description of reality.

But perhaps, Green argues, the connection to materials will show that people have fundamentally misunderstood what string theory is. "Maybe string theory is not a unique theory of reality, but something deeper - a set of mathematical principles that can be used to relate all physical theories," says Green. "Maybe string theory is the new calculus."

\section{Zeeya Merali is a freelance writer based in London}

\section{Maldacena, J. M. Adv. Theor. Math. Phys. 2, 231-252 (1998).}

2. Policastro, G., Son, D. T. \& Starinets, A. O. Phys. Rev. Lett. 87, 081601 (2001)

3. Luzman, M. \& Romatschke, P. Phys. Rev. C 78, 034915 (2008).

4. Herzog, C. P., Kovtun, P., Sachdev, S. \& Son, D. T. Phys. Rev. D 75, 085020 (2007).

5. Hartnoll, S. A., Kovtun, P. K., Müller, M. \& Sachdev, S. Phys. Rev. B 76, 144502 (2007)

6. McGreevy, J. Physics 3, 83 (2010)

7. Sachdev, S. Preprint at http://arxiv.org/abs/1108.1197v1 (2011)

8. Donos, A. \& Gauntlett, J. J. High Energy Phys. JHEP03(2009)138 (2009). 